\title{
The Influence of Pressure on the Roughness of InGaP Layers
}

\author{
E. Dumiszewska ${ }^{a}, \mathrm{P} . \mathrm{Knyps}^{a, b}, \mathrm{M}$ Wesolowski $^{a}, \mathrm{M}$ TeOdorczyK $^{a}$ And W. Strupinski ${ }^{a}$ \\ ${ }^{a}$ Institute of Electronic Materials Technology, Wólczyńska 133, 01-919 Warsaw, Poland \\ ${ }^{b} \mathrm{IMiO}$, Warsaw University of Technology, Koszykowa 75, 00-662 Warsaw, Poland
}

Today, the technology of typical silicon-solar cells is fully developed and mature. In spite of its continuous improvements, the record efficiencies of $25.0 \%$ are approaching theoretical solar conversion limits of around $33.7 \%$. Values much beyond this limit are likely to be achieved using III-V semiconductor compounds, electrical and optical properties are more suitable for solar energy conversion. They are the most promising candidates for realizing solar cells, which can achieve efficiencies of $50 \%$ and more. In this paper we studied the influence of pressure in the reactor chamber on the roughness of an InGaP "nucleation layer" grown on Ge. The growth of the layers was performed in a metalorganic vapour phase epitaxy reactor AIX 200/4. The source gases were trimethylgallium, trimethylindium and $\mathrm{AsH}_{3}$. The rate of pressure in the reactor was raised from $100 \mathrm{mbar}$ to 400 mbar by 50 mbar. The InGaP layers with the lowest roughness were achieved at the pressure of $400 \mathrm{mbar}$. The layers were characterized by very low roughness (RMS $<0.3$ ) measured by atomic force microscopy. The quality of the surface was perfect enough to be applied in a solar cell structure.

PACS: 77.55.dj, 78.30.Fs, 73.61.Ey, 81.10.-h

\section{Introduction}

III-V semiconductor compounds, whose electrical and optical properties are suitable for solar energy conversion are the most promising candidates for realizing solar cells, which can achieve efficiencies of $50 \%$ and more [1]. Such solar cells consist on three or more junctions (typically $\mathrm{InGaP} / \mathrm{InGaAs} / \mathrm{Ge}$ ) connected in series. Each of the junction absorbs different part of the solar spectrum in order to increase the efficiency and is called triple junction solar cell (TJ) [2]. To achieve the TJ with the best possible efficiency one has to optimize the performance of each junction. Unfortunately, there are many problems with achieving $\mathrm{Ga}_{0.5} \operatorname{In}_{0.5} \mathrm{P}$ (InGaP) layer on Ge substrate with the crystal quality and surface morphology perfect enough to be applied in solar cell structure. InGaP serves as the "nucleation layer" which provides defect-free template for subsequent epitaxial growth $[3,4]$. It should be characterized by as low surface roughness as possible. Here in this work we studied the influence of the lowering of the pressure in the reactor on the roughness of InGaP layer.

\section{Experiment}

All the growth processes were carried out in AIX 200/4 reactor. The growth of $\mathrm{InGaP}$ layers doped with silicon (so-called "nucleation layers") were carried out on germanium. The details of the growth process were published elsewhere [5]. The pressure in the reactor was varied from 200 to 400 mbar for the purpose of achieving InGaP layers with as low surface roughness as possible. The roughness of achieved layers were studied by means of atomic force microscopy (AFM).

\section{Results and discussion}

Our results show that the rising of the pressure in the reactor influences the roughness of the grown layers. Figures from 1 to 4 show the surfaces of the InGaP layers grown at the pressures from 250 to 400 mbar. The layer grown at the highest pressure is characterized by the lowest roughness.
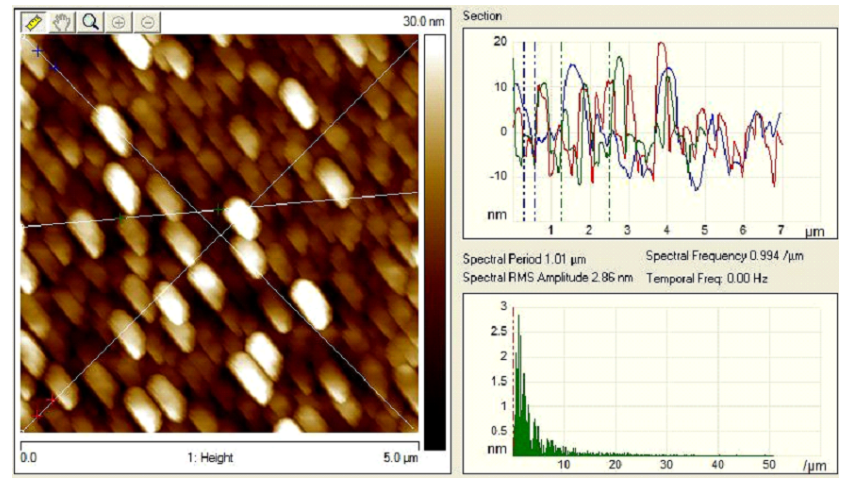

Fig. 1. The surface and roughness of InGaP layer grown in 250 mbar measured by AFM.
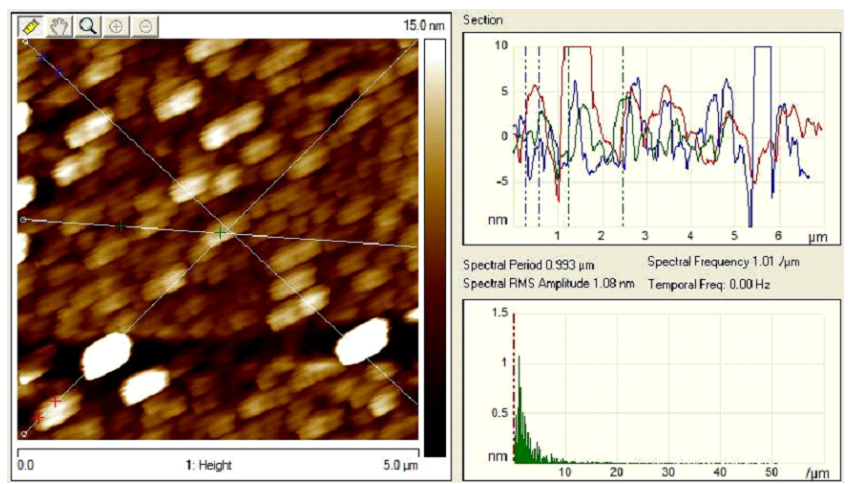

Fig. 2. The surface and roughness of InGaP layer grown in 300 mbar measured by AFM. 


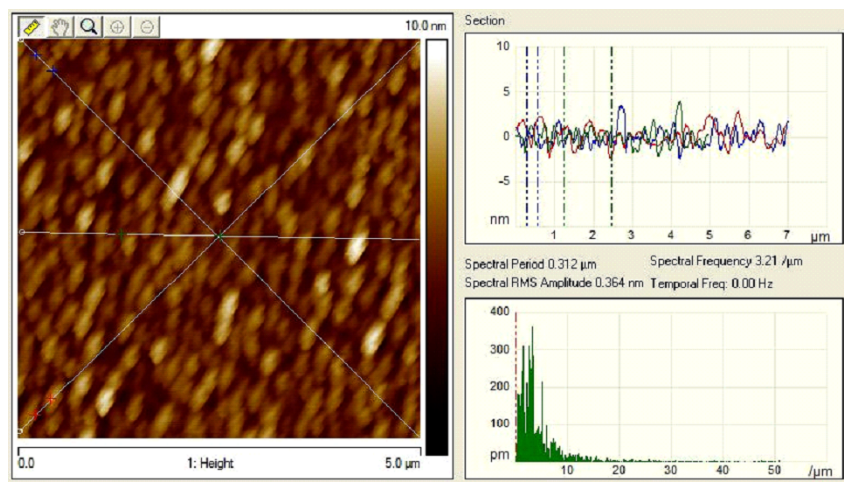

Fig. 3. The surface and roughness of InGaP layer grown in 350 mbar measured by AFM.

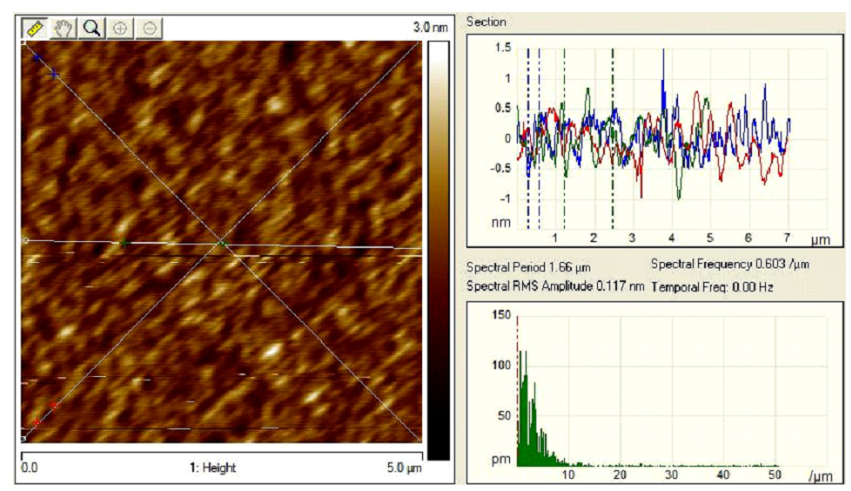

Fig. 4. The surface and roughness of InGaP layer grown in 400 mbar measured by AFM.
As a result of lowering the pressure in the reactor, the growth mode changed from three- to two-dimensional and, hence, the layer grown at 400 mbar is characterized by the lowest roughness and can be used as a template for the solar cell structure.

\section{Summary}

We investigated the influence of the pressure in the reactor on the roughness of $\mathrm{InGaP}$ layer. The rising of it made the growth mode change from 3 to $2 \mathrm{D}$ and hence the layer grown in the highest pressure was characterized by the lowest roughness and can be used as the template for solar cell structure.

\section{References}

[1] J.M. Olson, S.R. Kurtz, A.E. Kibbler, P. Faine, Appl. Phys. Lett. 56, 623 (1990).

[2] G.T.C. Flores, R. Campesato, Cryst. Res. Technol. 40, 1043 (2005).

[3] W. He, S.L. Lu, J.R. Dong, Y.M. Zhao, X.Y. Ren, K.L. Xiong, B. Li, H. Yang, H.M. Zhu, X.Y. Chen, X. Kong, Appl. Phys. Lett. 97, 121909 (2010).

[4] B. Galiana, E. Barrigón, I. Rey-Stolle, V. Corregidor, P. Espinet, C. Algora, E. Alves, Superlatt. Microstruct. 45, 277 (2009).

[5] E. Dumiszewska, P. Knyps, M. Wesolowski, M. Teodorczyk, W. Strupiński, in: EW-MOVPE XIV, Wrocław 2011, Extended Abstracts, D.04, Politechnika Wrocławska, Wrocław 2011. 